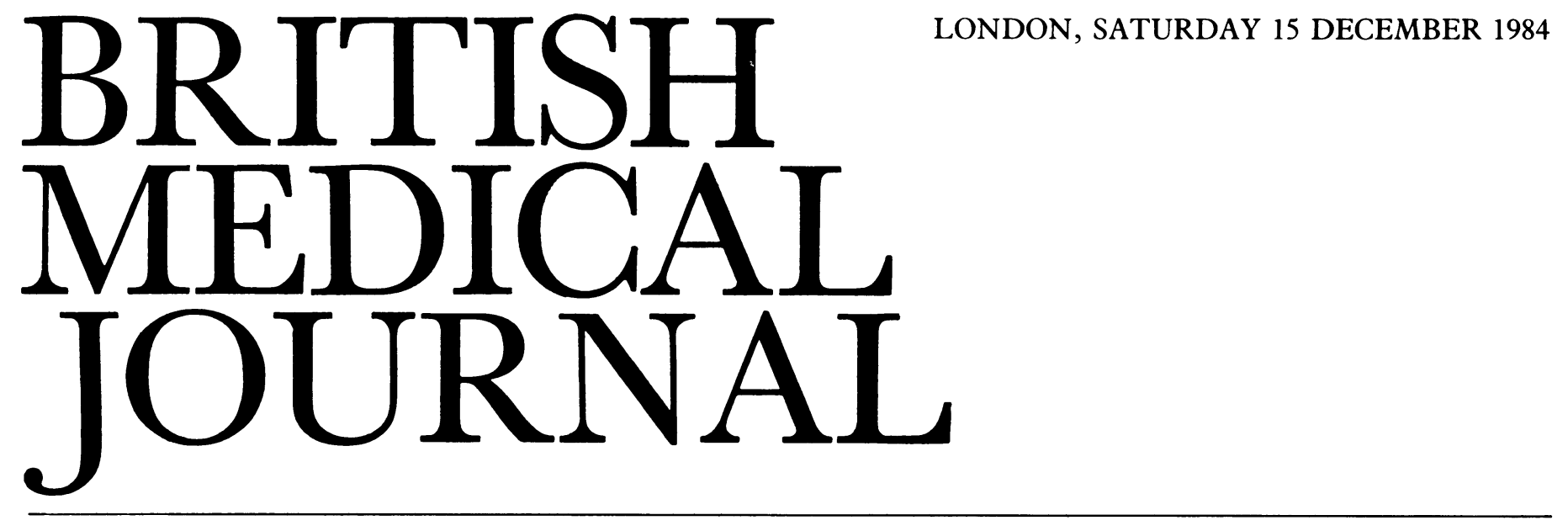

\title{
Why measure cholesterol after myocardial infarction, and when?
}

The value of measuring the serum cholesterol concentration after a patient has had a myocardial infarction depends on whether the subsequent prognosis is related to cholesterol or abnormal lipoprotein concentrations and whether lowering the raised serum lipid values improves this prognosis.

The prognosis after myocardial infarction is determined very largely by the extent of the myocardial damage sustained and the residual impairment of myocardial function. Not surprisingly, therefore, there are three studies which are consistent in finding no relation between plasma cholesterol concentrations measured after myocardial infarction and prognosis. Thus the Health Insurance Plan of New York, which reported the relation of serum cholesterol concentrations to prognosis in 437 patients with infarcts and 263 patients with angina, showed that the three and a half year mortality was actually worse in men with serum cholesterol concentrations below rather than above $7.0 \mathrm{mmol} / \mathrm{l}(270$ $\mathrm{mg} / 100 \mathrm{ml})^{\prime}$; a Canadian study showed no relation between serum cholesterol or low density lipoproteins $\left(S_{\mathrm{f}} 12-20\right)$ and survival in a good risk group of 120 men 10 years after myocardial infarction ${ }^{2}$; and Proudfoot $e t$ al in a study of 601 patients with angina or myocardial infarction also showed no relation between plasma lipid concentrations and 20 year prognosis, indicating its dependence on the extent of obstructive coronary artery disease and impairment of ventricular function. ${ }^{3}$

Apparently, the only study where a weakly positive association has been found between serum cholesterol concentration and prognosis after myocardial infarction is the Coronary Drug Project, with its 2789 male survivors aged 30-64. ${ }^{4}$ But this analysis of prognosis included 40 baseline variables assessed in a stepwise linear regression analysis, and cholesterol was the ninth variable selected. The first eight were all related to the state of the myocardium and arterial vasculature: these were ST segment depression, cardiomegaly, New York Heart Association function class, heart rate, ventricular conduction defects, history of diuretic use, number of myocardial infarctions, and history of intermittent claudication. While, a weakly positive overall relation was observed between cholesterol and prognosis, this resulted only from com- parison of the prognosis of those with initial serum cholesterol concentrations in the lowest quintile (median $5.1 \mathrm{mmol} / \mathrm{l} ; 195 \mathrm{mg} / 100 \mathrm{ml}$ ) with those in the highest quintile (median $8.3 \mathrm{mmol} / \mathrm{l} ; 320 \mathrm{mg} / 100 \mathrm{ml}$ ): thus a male survivor of myocardial infarction with a serum cholesterol concentration of $7 \cdot 8 \mathrm{mmol} / \mathrm{l}(300 \mathrm{mg} / 100 \mathrm{ml})$ had a five year probability of dying of $23 \%$ compared with $18 \%$ for a man with a concentration of $5 \cdot 2 \mathrm{mmol} / 1(200 \mathrm{mg} / 100 \mathrm{ml})$. No relation was found between prognosis and cholesterol at intermediate values, where most patients lie. The average length of time after myocardial infarction when baseline serum cholesterol concentrations were estimated was three years, while that of the negative studies was three to six months; thus the Coronary Drug Project study was conducted in late survivors, when the confounding factors of impaired myocardial function would be expected to be less. No relation was found between serum triglyceride concentrations and prognosis in any of these studies.

The answer to the question whether intervention to lower serum cholesterol concentrations has improved the prognosis after myocardial infarction is still unresolved. The same Coronary Drug Project showed that there was no reduction in coronary mortality after five years of lowering serum cholesterol concentrations by nicotinic acid or clofibrate, although there was a marginal $(p<0.05)$ reduction in the incidence of non-fatal myocardial infarction in those given nicotinic acid. ${ }^{5}$ In those with initial serum cholesterol concentrations above $7 \cdot 3 \mathrm{mmol} / \mathrm{l}(280 \mathrm{mg} / 100 \mathrm{ml})$ serum cholesterol was lowered below that of the placebo group on average by $7.5 \%$ or more in the clofibrate group and by about $10 \%$ or more in the nicotinic acid group, yet even in this subgroup with high initial values the mortality was not decreased. The Scottish and Newcastle trials using clofibrate showed no definite improvement in mortality after infarction; there was a significant positive effect in patients with angina, although it was not possible to relate this to the degree of consistency of lowering of serum cholesterol concentrations. ${ }^{6}$ Another study, not yet fully documented, has suggested that nicotinic acid does lower mortality and morbidity after the first myocardial infarction with commensurate lowering of cholesterol concentrations. ${ }^{7}$ The results of dietary trials after myocardial infarction are also conflict- 
ing with two negative small British studies ${ }^{89}$ and a positive open, non-blind Norwegian trial, where a low saturated fat and high polyunsaturated fat diet was reported to reduce the incidence of recurrent myocardial infarction and coronary deaths in men under 60 with initially high concentrations of serum cholesterol $(7 \cdot 7 \mathrm{mmol} / \mathrm{l} ; 296 \mathrm{mg} / 100 \mathrm{ml})$ : a $14 \%$ difference between the diet and control groups was achieved over the five years of this study. ${ }^{10}$ Without clearer, more convincing evidence of benefit we are not justified in imposing on all patients dietary or drug regimens which many find unpleasant.

Though measurement of serum cholesterol concentrations gives little guidance to prognosis and there is little to be gained from intervention for the average survivor of infarction, detection of hypercholesterolaemia is important. Its recognition should lead physicians to search for families with polygenic, or even perhaps the rare monogenic, hypercholesterolaemia. Families of patients who have had a myocardial infarction are likely to be well motivated to permit screening of all first degree relatives in order to improve their prognosis, and those with severe hypercholesterolaemia are likely to accept vigorous control measures. ${ }^{11}$ The most satisfactory way of doing this at present is to combine a diet with a high polyunsaturated to saturated fat ratio with $24-30 \mathrm{~g}$ cholestyramine daily. ${ }^{12}$

For these reasons it is helpful to be reminded that measurement of cholesterol within the first 24 hours after a myocardial infarction is a reliable way of assessing serum concentrations ( $p$ 1651). Contrary to a common belief, three months do not have to pass before an evaluation of plasma lipid values is possible. ${ }^{13}$ Serum concentrations of both cholesterol and low density lipoproteins decrease, however, during the second 24 hours and remain relatively low during the next two to three weeks. This is a message of practical value since blood is likely to be taken during the first 24 hours of myocardial infarction for various other biochemical measurements and less likely to be taken routinely three months after the heart attack. Estimation of serum cholesterol concentrations should be routine during the first 24 hours in all young and middle aged patients with myocardial infarction in order to detect and treat those and their close relatives with concentrations around and above $8.0 \mathrm{mmol} / \mathrm{l}$ $(309 \mathrm{mg} / 100 \mathrm{ml}$ ).

\section{F OLIVER}

Duke of Edinburgh Professor of Cardiology,

Cardiovascular Research Unit,

University of Edinburgh,

Edinburgh EH8 9XF

1 Frank CW, Weinblatt E, Shapiro S. Prognostic implications of serum cholesterol in coronary heart disease. In: Jones RJ, ed. Atherosclerosis. Proceedings of the second intermational symheart disease. In: Jones Re, ed. Atheroscle

2 Shanoff HM, Little JA, Csima A. Studies of male survivors of myocardial infarction: XII. Relation of serum lipids and lipoproteins to survival over a 10-year period. Can Med Assoc $\mathcal{J}$ 1970;103:927-31.

3 Proudfoot WL, Bruschke AVG, Sones FM. Natural history of obstructive coronary artery disease: ten-year study of 601 nonsurgical cases. Prog Cardiovasc Dis 1978;21:53-78.

4 Coronary Drug Project Research Group. Natural history of myocardial infarction in the Coronary Drug Project: long-term prognostic importance of serum lipid levels. Am $\mathcal{J}$ Cardio

Coronary Drug Project Research Group. Clofibrate and niacin in coronary heart disease. JAMA Dewar HA, Oliver

Newcastle and Scottish trials. Br Med $\mathcal{J} 1971$;iv: $784-6$.
Newar HA, Oliver MF. Secondary prevention trials using

Newcastle and Scottish trials. Br Med 7 1971;iv:784-6. heart disease. Atherosclerosis 1980;37:129-38.

8 London Hospitals Research Committee. Low fat diet in myocardial infarction. A controlled clinical trial. Lancet 1965;ii:501-4.

Rose GA, Thomson WB, Williams RT. Corn oil in treatment of ischaemic heart disease. $\mathrm{Br} \mathrm{Med}$

J $1965 ;$; i: $1531-3$.
10 Leren $P$. The effect of plasma cholesterol lowering diet in male survivors of myocardial infarction. Acta Med Scand 1966; suppl 466

1 Oliver MF. The case for intervention in those at high risk for coronary heart disease. Advanced

1984;288:423-4.

13 Fyfe T, Baxter RH, Cochran KM, et al. Plasma lipid changes after myocardial infarction. Lance 1971;ii:997-1001.
Aseptic rituals unmasked

Debunking venerable medical customs and procedures is a beguiling pastime which may have practical as well as recreational value. Among the most attractive targets are the surgical rituals introduced by Joseph Lister and his direct disciples. The recent advances in our knowledge of the microbiology of the skin, mucous membranes, and gut have stimulated reappraisal of the measures designed to protect the patient from infection. Traditionally these have included the surgical scrub, as well as shaving, disinfecting, draping, and subsequently dressing the operation site, and also preparing the bowel before surgery on the gut.'

Probably the most visually impressive feature of aseptic ritual is the cocooning of staff in bizarre gowns, hats, and masks. At one extreme a complete physical barrier may be donned by the surgical team in the form of the body exhaust ventilated "space suit," which is an integral part of the Charnley-Howorth system of ultraclean air in operating rooms. This degree of protection of the patient from the staff's microflora may be justified only in high risk procedures such as replacement of the hip joint. ${ }^{2}$ The more conventional types of operating costume form a negligible barrier to micro-organisms unless the normal woven fabrics are replaced by impermeable - and generally uncomfortable - materials. ${ }^{3}$

At first glance facemasks may seem a small and unimportant part of the protective uniform. They can, however, prevent the direct contamination of the operation field with respiratory tract droplets containing pathogenic bacteria. In obstetric practice this appears to be a less frequent source of infection than the patient's own microflora. In one recent study, for example, staff stopped wearing masks during vaginal delivery and the incidence of puerperal infection in 1750 consecutive cases $(3.6 \%)$ was the same as in the previous 1750 cases when masks had been worn. ${ }^{4}$ The wearing of masks was continued for all caesarean sections, yet a quarter of the 192 patients developed puerperal infections, which were apparently endogenous in most cases.

In this study, fortunately, no infections occurred with Lancefield group A haemolytic streptococci, but nevertheless four of the five infections with "streptococcal species" (other than group A or B) were in patients attended by unmasked staff. For almost 200 years the principal danger facing women during labour has been known to be infection acquired from their attendants. ${ }^{5}$ The arch enemy, the haemolytic streptococcus, is harboured in the upper respiratory tract of about $1 \%$ of the general population ${ }^{6}$ and is transmitted to the birth canal infrequently but with potentially disastrous consequences. ${ }^{78}$

This risk is far less than 50 years ago in the heyday of puerperal fever, when around one fifth of the population were carriers. The high incidence at that time helped to establish clearly the importance of droplet borne infection from staff, notably in a classic $B M \mathcal{F}$ paper of $1935 . .^{9}$ This included impressive epidemiological data to show "that genital infection is not homo-exogenous but heteroexogenous-that is, from the respiratory tract of obstetric attendants." The paper also described pioneer experiments on the protective value of masks in obstetrics and surgery. An efficient mask must be capable of arresting "low momentum droplets," which contaminate "the front of the operator's gown and his sterile gloves, transference to the patient occurring at the next manual interference," as well as the 